\title{
Emugel for topical drug delivery: A novel approach
}

\author{
Devi Suman ${ }^{1}$, Sangeeta ${ }^{2}$ and Kumari Beena ${ }^{2, *}$ \\ ${ }^{1}$ SDM College of Pharmacy, Rajound. Kaithal. \\ ${ }^{2}$ Department of Pharmaceutical Sciences, Indira Gandhi University, Meerpur, Rewari, Haryana, India-123401.
}

Publication history: Received on 06 June 2020; revised on 16 June 2020; accepted on 18 June 2020

Article DOI: https://doi.org/10.30574/gscbps.2020.11.3.0165

\begin{abstract}
Over the last decades the treatment of disease has been accomplished by administering drug to human body via various routes namely oral, sublingual, rectal and parental etc. The topical drug delivery system generally used when the systems of drug administration fails or in local infection like fungal infection. The topical application of the drug offer the potential advantages for delivering the drug directly to the site of action and delivering the drug for extended period of time at the affected site that mainly acts at the related region. Topical drug delivery can be defined as the application of a drug containing formulation to the skin to directly treat cutaneous disorder e.g. acne or the cetaceous disorder. The various groups of semisolid preparations, the use of gels has expanded both in cosmetics and in pharmaceutical preparations. Despite of several advantages of gels there is a major limitation in delivery of hydrophobic drug moiety. This limitation can be overcome by the use of novel topical drug delivery i.e. Emulgel. Emulgel is one of the recent technologies in NDDS used topically having characteristics of dual control release i.e. emulsion as well as gel. Emulgel are having major advantages on novel vesicular systems as well as on conventional systems in various aspects. Emulgel for dermatological use have several favorable properties such as being thixotropic, greaseless, easily spreadable, emollient, non-staining; water soluble, longer shelf life, bio-friendly, transparent and pleasing appearance.
\end{abstract}

Keywords: Emulgel; Topical drug delivery; Skin; Gelling agent; Evaluation.

\section{Introduction}

The field of pharmaceutical science has been developing steadily over the years and now a days it become invaluable in helping to keep us healthy and prevent disease. An avenue of research that has progressed a great deal in the past few decades in the treatment of diseases via biomolecules such as drugs, proteins etc. Over the last decades the treatment of disease has been accomplished by administering drug to human body via various routes namely oral, sublingual, rectal, parental etc. [1]. The topical drug delivery is generally used where these systems of drug administering fails or in local skin infection like fungal infection. Now a day many dosage forms are given by oral and parental. Oral route is widely used but it follows GI-side effects, first-pass metabolism and results decrease in the bioavailability of the drug. In such cases, parental preparations are better than oral preparations requires well equipped laboratory with aseptic area. These make parental costlier [2]. The goal of any drug delivery system is to provide a therapeutic amount of drug to the proper site in the body to promptly achieve and then maintain the desired drug concentration. One of such drug delivery system used since ancient time is Topical drug delivery system (TDDS) [3]. Topical drug delivery can be defined as the application of a drug containing formulation to the skin to directly treat cutaneous disorder. Topical drug administration is localized drug delivery system anywhere in the body through ophthalmic, rectal, vaginal and skin as topical routes. These are applying a wide spectrum of preparations for both cosmetic and dermatological, to their healthy or diseased skin. Skin is one of the most readily accessible organs on human body for topical administration and is main route for topical drug delivery system. Dermatological products are diverse in formulation from liquid to powder but most popular products are semisolid preparation. Topical drug delivery is an attractive route for local and systemic

\footnotetext{
${ }^{*}$ Corresponding author: Kumari Beena
} 
treatment [4]. Topical drug delivery offers several advantages over conventional routes. It avoids the first-pass metabolism and the gastrointestinal tract. Topical delivery has the potential for sustained and controlled drug release. It is noninvasive mode of drug delivery with no risk of infection. The most common example of topical dosage forms includes solutions, suspension, emulsion, semisolids (e.g. foams, ointments, pastes, creams and gels), solids and sprays [5].

Within the major group of semisolid preparation, the use of transparent gels has expanded both in cosmetics and in pharmaceutical preparations. Gels are newer class of dosage form created by entrapment of large amounts of aqueous or hydro alcoholic liquid in a network of colloidal solid particles. In spites of many advantages offered by gels a major limitation is in the delivery of hydrophobic drugs. So this limitation can be overcome by using an emulsion based approach where gels and emulsions are combined to form Emulgel [6]. Both oil-in-water and water-in-oil emulsions are used as vehicles to deliver various drugs to the skin. In recent years, there has been great interest in the use of novel polymers with complex functions as emulsifiers and thickeners because the gelling capacity of stable emulsions and creams by decreasing surface and interfacial tension and at the same time increasing the viscosity of the aqueous phase [7]. The presence of gelling agent in the water phase converts a classical emulsion into Emulgel. Emulgel for dermatological use have several favorable properties such as being thixotropic, greaseless, non-staining, easily spreadable, easily removable, emollient, long shelf life, transparent and pleasing appearance $[8,9]$.

\subsection{Rational}

Emulgel emerges as one of the important and better way of delivering the drug; it is because of its better control ability over the other topical dosage forms. Many widely used topical agents like ointment, cream and lotion have many disadvantages i.e. very sticky causing uneasiness to the patient when applied. They have lesser spreading coefficient and need to apply with rubbing and they exhibit the stability problem. Due to all these factors within the major group of semisolid preparations, the use of transparent gels has expanded both in cosmetics and in pharmaceutical preparations. A gel is colloid that is typically $99 \%$ weight liquid, which is immobilized by surface tension between it and a macromolecular network of fibers built from a small amount of gelatin substance present. In spite of many advantages a major limitation is in delivery of hydrophobic drugs. So to overcome this limitation an emulsion based approach is being used so that a hydrophobic therapeutic moiety can be successfully incorporated and delivered through gels. The Emulgel preparations are much more stable than that of other types of topical preparation like powder may be hygroscopic that may absorb environmental humidity during its direct exposure to the surroundings, creams shows phase inversion and the ointment shows rancidity due to oil base.

\section{Classification of Topical preparation}

Topical drug delivery systems are classified on the basis of their formulation. Various classes of Topical drug delivery system (TDDS) are classified in figure 1.

\subsection{Factors affecting topical absorption of drug $[10,11]$ :}

There are some factors which affect the absorption of drugs. Few of them are listed below.

\subsubsection{Physiochemical Factors}

- Partition coefficient

- Molecular weight (<500 Dalton)

- Degree of ionization

- Effect of vehicles

\subsubsection{Physiological Factors:}

- Skin thickness

- Density of hair follicles

- Density of sweat gland

- Blood flow

- Hydration of skin

- Inflammation of skin

- Type of skin

- Skin $\mathrm{pH}$

- Lipid contents 


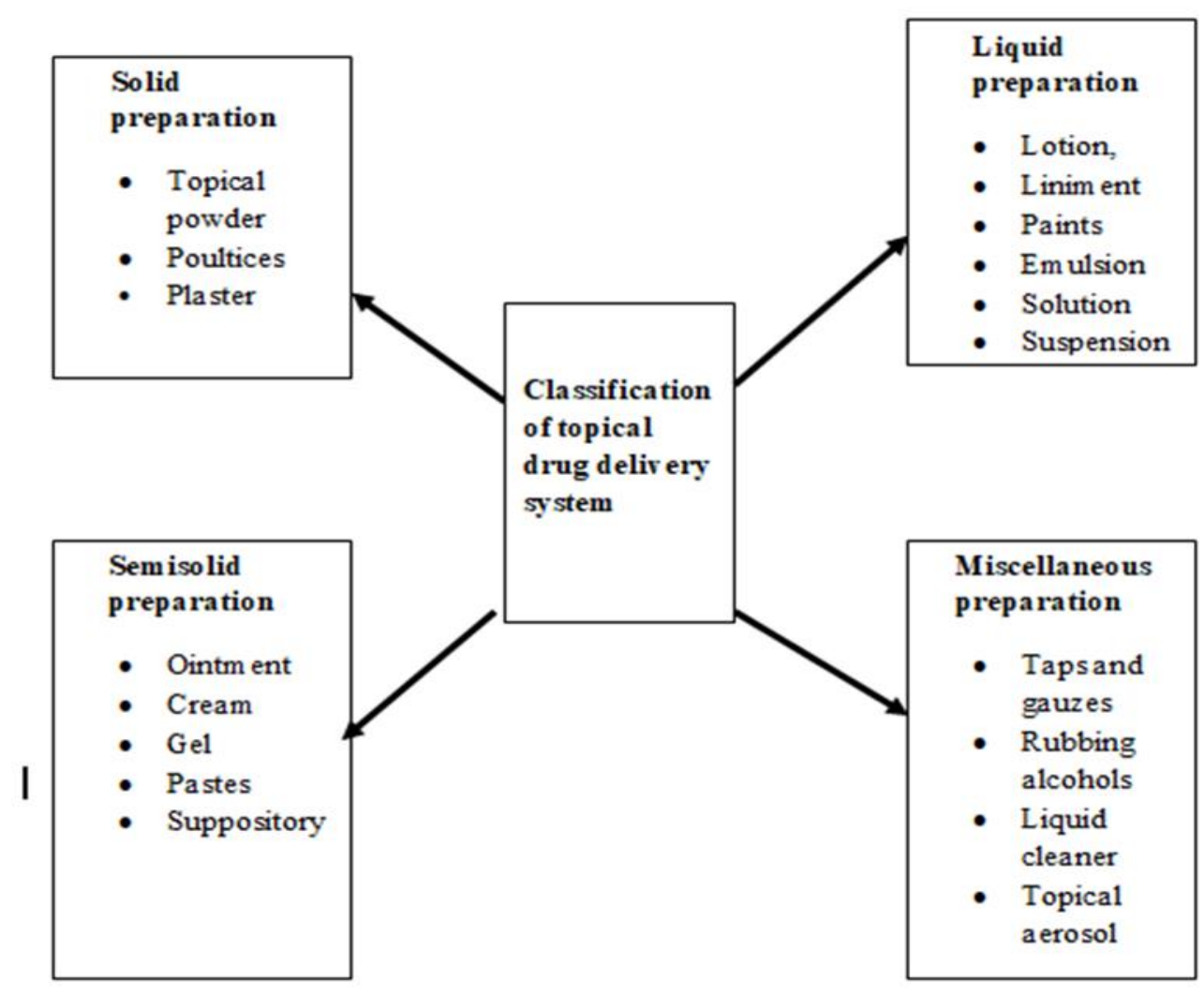

Figure 1 Classification of topical drug delivery system

\subsection{Physiology of Skin [12-14]}

Skin is the major organ for Topical Drug Delivery System (TDDS) and molecules penetrate into the skin by three routes: through stratum corneum, sweat ducts and sebaceous follicle. The surface area of the adult skin is approximately $2 \mathrm{~m}^{2}$ and it receives one third of the blood circulating through the body. The human skin contains about 40-70 hair follicles and 200-300 sweat ducts. The $\mathrm{pH}$ of the human skin varies from 4 to 5.6 .

\subsection{Classification of Skin layers}

Skin layers may be classified into three parts:

- Epidermis

- Dermis

- Subcutaneous tissue

\subsubsection{Epidermis layer}

Epidermis is the outer layer of skin and it is approximately $150 \mu \mathrm{m}$ thick. Cells from the lower layers of the skin travel upward during their life cycle and they become flat dead cell of the corneum. The energy source for the lower portion of the epidermis skin is the Glucose and lactic acid which is end product of the metabolism.

The layers of Epidermis are

- Stratum Germinativum (Growing Layer)

- Malpighion Layer (pigment layer)

- Stratum Spionsum (Prickly cell Layer)

- Stratum Granulosum (Granular Layer)

- Stratum Lucidum (false layer)

- Stratum Corneum (horny Layer) 


\section{Stratum Germinativum/ Stratum basal}

It is known as the growing layer because the cell of this layer have high mitotic index and constantly renew the epidermis. Te healthy skin balances the loss of dead horny cell from the skin surface. This is single cell layer.

\section{Malpighion Layer}

It is known as the pigment layer. The basal cell include melanocytes which produce the distribute melanin granules to the keratinocytes required for pigmentation a protective measure against UV radiation.

\section{Stratum spinosum}

The cell of the layer is produce by morphological and histochemical alteration of the basal layers as they moved upward. The cells flatten and their nuclei shrink. They are interconnected by fine prickles, so it is known as prickly cell layer. These links maintain the integrity of the epidermis.

\section{Stratum Granulosum}

This layer is above the keratinocytes, because the presence of keratinohylline granules, so it is known as granular layer. This keratogenous zone is a region of intense biochemical activity and morphological change.

\section{Stratum Lucidum}

It is a thin, clear layer of dead skin cells in the epidermis. It is readily visible by light microscopy only in the areas of thick skin, which are found on the palm of the hand and sole of the feett and forms a thin translucent layer immediately. The cells are non-nuclear. It is composed of three to five layers of dead, flattened keratinocytes. The keratinocytes of the stratum lucidum do not feature distinct boundaries and it an intermediate form of keratin. The thickness of lucidum is controlled by mitosis.

\section{Stratum corneum}

It is the superficial layer of the epidermis. It is the physical barrier to most substance that comes in contact with the skin. The thickness of the stratum corneum is 10 to 20 cells. Each cell is flat, plate like structure and 34-44 $\mu \mathrm{m}$ long, 25$36 \mu \mathrm{m}$ wide, 0.5 to $0.20 \mu \mathrm{m}$ thick with surface area of 750 to $1200 \mu \mathrm{m}$ stocked up to each other in brick like fashion. It consists of lipid (5-15\%) including phospholipids, glycosphingo lipid, cholesterol sulfate and protein (75-85\%) which is mainly keratin. The cell of this layer contains mainly keratin, so these cells are also known as Coreanocytes.

\subsubsection{Dermis}

It consists mainly of the dense network of structural protein fibers, i.e. collagen, reticulum, and elastin, embedded in the semigel matrix of mucopolysaccaridic 'ground substance'. The elasticity of the skin is due to the gel structure of the cells. The thickness of the dermis layer is 2000 to $3000 \mu \mathrm{m}$ and consists of a matrix of connective tissue. The dermis layer support and interacts with the epidermis and facilitating to the underlying muscles and bones.

\subsubsection{Subcutaneous tissue}

It consists of sheet of fat rich areolar tissue known as superficial fascia, attaching the dermis to the underlying structure. Large arteries and vein are present in the superficial region.

\subsection{Permeation of the drug through the skin $[15,16]$}

The skin forms a waterproof layers that protects the deeper and more delicate structures. There are two important layers in the skin: the dermis and epidermis. Epidermis is the most superficial layers of the skin and is composed of stratified keratinized epithelium which varies in thickness in different part of the body. The thickness of the epidermis layers is approximately 100 to $150 \mu \mathrm{m}$ and has no blood flow. The layer include within the epidermis is stratum corneum. The thickness of the stratum corneum is approximately 10 to $15 \mu \mathrm{m}$ and is mainly consist of dehydrating and dead keratinocytes. There are three mechanisms of topical drug absorption: transcellular, intercellular and follicular. The most common route of drug delivery is the pilosebaceous route. Mostly drugs are absorbed by passive diffusion and if drug able to penetrate the stratum corneum then it can enter into the blood stream. The reservoir and matrix are the two concepts in the design of transdermal drug delivery system. Both involve diffusion diffusion of drug through the skin. The enhancement effect required for the delivery of low permeability coefficients macromolecules and the capability of chemical enhancer tolerated by skin. Modulation of formulation excipients and addition of permeation enhancers, such as fatty acid, surfactant, esters and alcohols that exert their action by a temporary alteration of barrier 
properties of the stratum corneum by various mechanism; including enhancing solubility, partitioning the stratum corneum, fluidizing the crystalline structure of the stratum corneum and dissolution of stratum lipids enhance the drug flux.

Gels and creams that are rubbed into the skin have been used for years to deliver medication and infection fighting drug to an affected site of the body. New technologies now allow other drug to be absorbed through the skin. These can be used to treat not just the affected areas but the whole body.

\section{Advantages of Emulgel $[17,18]$}

- Hydrophobic drug can be easily incorporated by using o/w emulsion.

- Better stability.

- Better loading capacity.

- Low preparation cost.

- Prolonged effect of drug (controlled release).

- Improve patient compliance.

- Avoidance of first pass metabolism.

- Self-applied medication.

- Termination of therapy when required.

- Suitable for drug with short half-life and for potent drug.

- Site specific drug delivery system.

- Avoidance of gastrointestinal incompatibility.

\section{Formulation Methods}

There are some methods of preparation of Emulgel using different kinds of ingredient, by various researchers. One method reported by Mohamed (2004) in his research work (optimization of chlorphenesin in Emulgel)

Which includes formation of emulsion (o/w or w/o), followed by addition of gelling agent to form Emulgel. Emulsion was prepared by dissolving tween 20 in purified water and solution of propylene glycol was prepared by dissolving methyl paraben and propyl paraben in propylene glycol after that both the solution were mixed and set aside. The oily phase of emulsion was prepared by dissolving span 20 in light liquid paraffin. Both aqueous and oily phase were separately heated at $70-80{ }^{\circ} \mathrm{C}$ and then both the phases were mixed with constant stirring until cooled at room temperature. Gel phase of the Emulgel was prepared by dispersing HPMC or Carbopol in water. HPMC was required to soak overnight in water, while Carbopol gel was prepared by simply dispersing it in purified water. When both the component both emulsions and gel get ready then Emulgel was prepared by mixing emulsion with gel in 1:1 ratio with gentle stirring [19].

Another method was reported by Perioli et al. (2008). In this research formulation using three steps for the preparation of Emulgel (i) polymer dispersion in water, (ii) neutralization of the polymeric aqueous dispersion and (iii) emulsification of oil phase. With respect to the first step, three different TR- 1 percentages, namely $0.3,0.4$ and 0.5 , w/v, was required. First step involves suspension of polymer in deionized water with continuous stirring at $900 \mathrm{rpm}$ for 20 minutes at room temperature using mechanical stirrer equipped with a three blade helical impellers and then slurry was neutralized with sodium hydroxide solution $(18 \% \mathrm{w} / \mathrm{v})$ to final $\mathrm{pH}$ value of $5.5,6.0$ and 6.5 . The neutralization process causes the distension of polymer chains resulting in clear stable gels. Now for the complete hydration of polymer gels were required to be stored at $4{ }^{\circ} \mathrm{C}$ for 24 hours before the addition of the oil phase. After completing the hydration of gel different quantities of oil phase at three $\mathrm{o} / \mathrm{w}$ ratios $(\mathrm{w} / \mathrm{w}) 0.5,1.0$ and 1.5 respectively were added with stirring at $800 \mathrm{rpm}\left(80^{\circ} \mathrm{C}\right)$ and then left for cooling and its $\mathrm{pH}$ was measured [20].

Shahin et al. (2011) followed a different method to develop Emulgel for clotrimazole delivery. This method involves the oily phase of emulsion by dissolving drug and span 60 in oily phase (jojoba oil) with the aid of magnetic stirrer at $75^{\circ} \mathrm{C}$ with subsequent cooling followed by addition of Carbopol to oily phase. In the second step the aqueous phase was prepared by dissolving Brij-35 in propylene glycol. In the third step addition of oily phase to the aqueous phase using the overhead mixer for 10 minutes at $1400 \mathrm{rpm}$ and then introducing emulsion into the homogenizer for 5 minutes at $10,000 \mathrm{rpm}$. Gellification of emulsion involves addition of gelling agent triethanolamine (formulae containing carbopol 
either alone or in combination) .and/or HPMC to the emulsion using overhead mixer at $200 \mathrm{rpm}$ for 45 minutes thereby adjusting the $\mathrm{pH}$ of formulation containing Carbopol to 5.5-6.5 using triethanolamine [21].

Dickinson Eric et al. (2012) prepared a solid- like emulsion gel from a stable liquid- like emulsion by gelling the continuous phase or aggregating the emulsion droplets. Simply forcing all the droplets closer together by centrifugation can be sufficient to produce a concentrated protein stabilized emulsion with gel-like behavior. Emulsion gel produce by heat treatment have limited uses, especially for food formulation containing heat sensitive ingredients. The appearance of the emulsion gels prepared by salt-induced gelation was highly dependent on calcium concentrations. Increasing salt concentration produced a particulate gel with poor water holding capacity [22].

\subsection{Method of preparation for Emulgel [23]}

Step1: Formation of emulsion either o/w or w/o.

Step2: Formation of gel base.

Step3: Incorporation of emulsion into gel base with continuous stirring (Fig 2).

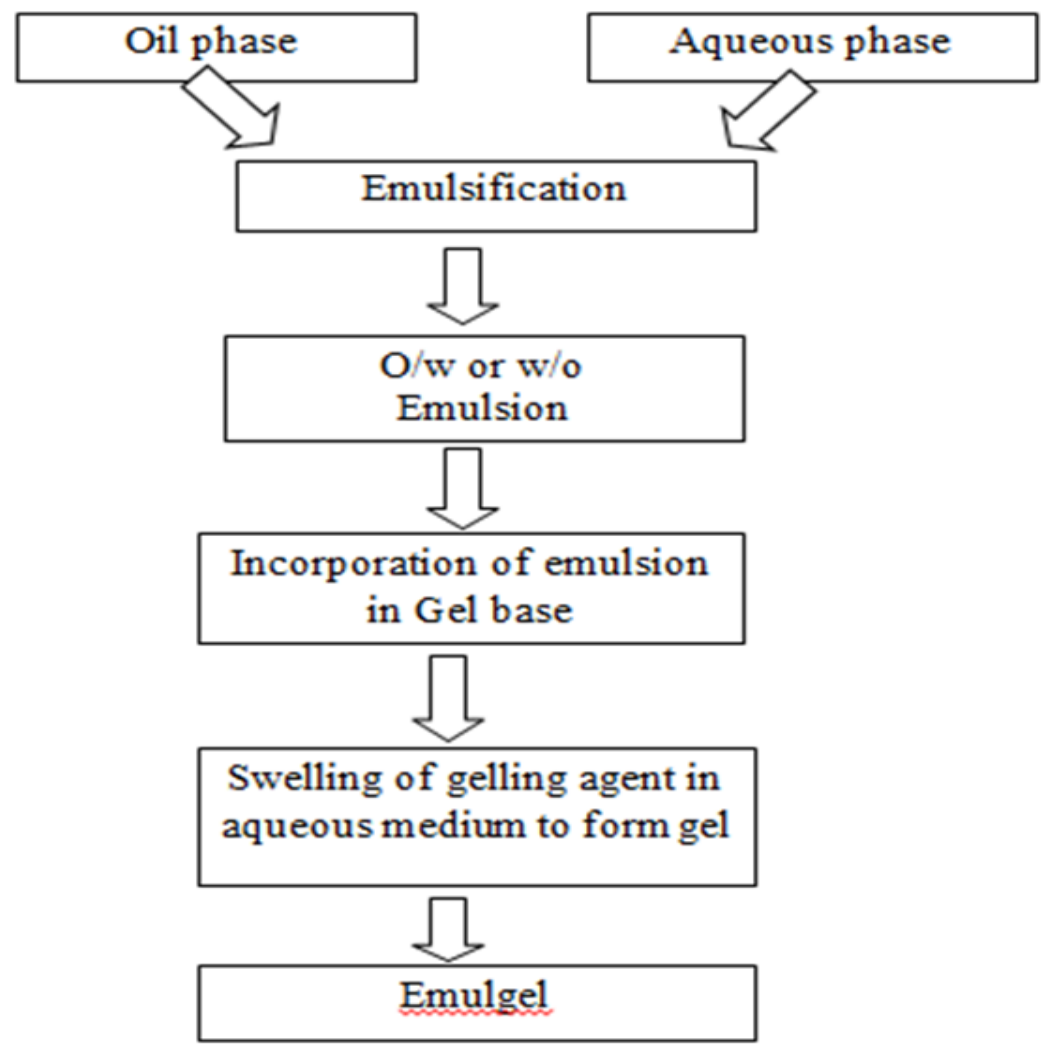

Figure 2 Flow chart for Emulgel

\subsection{Important constituents for the preparation of Emulgel}

Vehicle

- Aqueous material

- Oils

Emulsifiers

Gelling agents

Permeation enhancers 


\subsubsection{Vehicle}

The vehicle is an important link between drug potency and therapeutic effectiveness, since extensive pharmaceutical research has shown that the composition of vehicle can profoundly influence the rate and extent of absorption. Substance in the vehicles, such as humectants, which have high affinity for water, may under certain circumstances dehydrate the stratum corneum and decrease penetration. They also affect the penetration of drug by loss of water vapor on the skin surface [24]. The vehicle has following properties:

- Deposit the drug on the skin with distribution.

- The release of drug migrates freely at the site of action.

- Deliver the drug at the target site and sustain the drug release.

- Appropriately formulated for the anatomic site to be treated.

- Cosmetically acceptable to the patent.

Aqueous material

Aqueous material forms the aqueous phase of emulsion. The commonly used agents are water and alcohols etc [25].

Oils

Oils form the oily phase of the emulsion. For externally applied emulsion, non-biodegradable mineral castor oils that provide a local laxative effect, fish liver oils or various fixed oils of vegetable origin as nutritional supplement [26]. Other excipients (vehicles) used are given in table 1.

Table 1 Quantity of vehicles used in Emugel

\begin{tabular}{lll}
\hline Chemicals & Quantity & Dosage form \\
\hline Light liquid paraffin & $7.5 \%$ & Emulsion and Emulgel \\
Isopropylmyristate & $7-7.5 \%$ & Emulsion \\
Isopropyl stearate & $7-7.5 \%$ & Emulsion \\
Isopropyl palmitate & $7.7 .5 \%$ & Emulsion \\
Propylene glycol & $3-5 \%$ & Gel \\
\hline
\end{tabular}

\subsubsection{Emulsifier}

Emulsifying agent is mainly used for the promotion of emulsification of oil and aqueous phase at the time of formulation. They retard the phase separation of emulsion by increasing the stability and shelf life of emulsion can be vary from days to months or years for commercial preparation. Emulgel mostly contains Polyethylene glycol, Sorbitan mono-oleate (Span 80), Polyoxyethylene sorbitan monooleate (Tween 80), Stearic acid and sodium stearate as emulsifying agents [27].

\subsubsection{Gelling agents}

These agents are used to form gel base to incorporate emulsion in it to prepare Emulgel. Gelling agents are used to increase the consistency of any dosage form by swelling in aqueous phase and form gel like structure. These are used as thickening agent in Emulgel [28]. The examples of gelling agents are given in table 2.

Table 2 Quantity of Gelling Agents used in Emugel

\begin{tabular}{lll}
\hline Gelling agents & Quantity & Dosage form \\
\hline Carbopol-934 & $1 \%$ & Emulgel \\
Carbopol-940 & $1 \%$ & Emulgel \\
HPMC-2910 & $2.5 \%$ & Emulgel \\
HPMC & $3.5 \%$ & Gel \\
Sodium CMC & $1 \%$ & Gel \\
\hline
\end{tabular}




\subsubsection{Penetration enhancers}

These are the agents which are used to increase the penetration power of the drug through the skin. These promote the absorption of drugs through the skin and they temporarily disrupts the highly ordered structure of stratum corneum skin barrier, fluidized the lipid channels between corneocytes, alter the partitioning of the drug into the skin structures, or enhance delivery into skin [29]. Penetration promoting agents mostly used in topical drug delivery are given in Table 3.

Table 3 Quantity of Penetration Enhancer used in Emugel

\begin{tabular}{lll}
\hline Penetration Enhancer & Quantity & Dosage form \\
\hline Oleic acid & $1 \%$ & Emulgel \\
Lecithine & $5 \%$ & Gel \\
Urea & $10 \%$ & Gel \\
Isopropyl myristate & $5 \%$ & Gel \\
Linoleic acid & $5 \%$ & Gel \\
Clove oil & $8 \%$ & Emulgel \\
Menthol & $5 \%$ & Emulgel \\
\hline
\end{tabular}

\section{Evaluation parameters for Emulgel}

\subsection{Physical appearance}

The prepared Celecoxib Emulgel was inspected visually for their colour, consistency, and homogeneity and phase separation [30].

\section{2. $\mathrm{pH}$ determination}

The $\mathrm{pH}$ values of prepared Emulgel formulation were determined by using $1 \% \mathrm{w} / \mathrm{v}$ aqueous solutions of the prepared Emulgel by digital meter. One gram of Emulgel was dissolved in $100 \mathrm{ml}$ was dissolved in $100 \mathrm{ml}$ of distilled water and it was placed for two hours. The measurement of $\mathrm{pH}$ of each formulation was done in triplicate and average value was calculated [31].

\subsection{Spreadability study}

Spreadability coefficient was to be determined by apparatus suggested by Mutimer. The apparatus consists of wooden block, which is attached to a pulley at one end. Spreading coefficient was measured on the basis of 'Slip' and 'Drag' characteristics of prepared Emulgel. A ground glass slide having same dimension as that of the fixed ground slide was provided with the hook. Weight of $20 \mathrm{gm}$ was placed on the top of the two slides for 5 minutes to expel air and to provide a uniform film of Emulgel between the two slides. Measured quantity of weight was placed in the pan attached to the pulley with the help of hook. The time (in seconds) required by the top slide to separate from ground slide was noted [32]. The spreadability is calculated by using the Formula.

$$
S=\frac{M \cdot L}{T}
$$

Where $\mathrm{M}=$ wt. tied to upper slide.

$\mathrm{L}=$ Length of glass slide.

$\mathrm{T}=$ Time taken to separate the slides.

\subsection{Extrudability}

A $15 \mathrm{gm}$ of Emulgel was filled in aluminum tube. The plunger was adjusted to hold the tube properly. The weight of 1 $\mathrm{kg} / \mathrm{cm}^{2}$ was applied for 30 seconds the quantity of Emulgel extruded was weighed [33]. 


\subsection{Rheological study}

Viscosity of the prepared Emulgel was determined using digital viscometer (Labman LMDV-60 Digital Rotational Viscometer L2415) at the temperature of $37^{\circ} \mathrm{C}$. The formulation was added to the beaker, Spindle number III was lowered perpendicular in to the centre of Emulgel taking care that spindle does not touch bottom to the jar and rotated at a 10 (minimum) and 100 (maximum) rotations per minute, the viscosity was noted down [34].

\subsection{Drug content determination}

Drug concentration in Emulgel was calculated by spectrophotometric method. Celecoxib content in Emulgel was estimated by dissolving $1 \mathrm{gm}$ of Emulgel in $100 \mathrm{ml}$ solvent (methanol) by sonication (Ultrasonic Probe Sonicator). Aliquots of different concentrations were prepared by suitable dilution after sonication and filtering the stock solution and absorbance was measured $254 \mathrm{~nm}$ in UV-VIS spectrophotometer (Systronic PC Based Double Beam Spectrophotometer 2202). Drug content was calculated using the equation, which was obtained by linear regression analysis of calibration curve [35].

\subsection{In vitro drug release}

The in vitro drug release studied of the Emulgel was carried out on Franz diffusion cell using egg membrane. Take a fresh egg and put into the concentrated HCL for $15 \mathrm{~min}$. and then remove into the HCL and put into the fresh water for $5 \mathrm{~min}$. and then remove the egg membrane. This egg membrane was clamped carefully between donor and receptor compartment. Emulgel (1 gm) was applied on to the surface of egg membrane. The receptor chamber was filled with freshly prepared Phosphate buffer solution (5.5) to solubilize the drug the drug. The receptor chamber was stirred by magnetic stirrer. $5 \mathrm{ml}$ aliquots were withdraw at 10, 30, 60, 90, 120, 180, 240, 300, 360, 420, 480 minutes. The samples after filtrations were assayed spectrophotometrically at $254 \mathrm{~nm}$. Each determination was carried out in triplicate. Cumulative corrections were made to obtain the total amount of drug release at each time interval. The cumulative amount of drug release across the egg membrane was determined as a function of time [36].

\section{Conclusion}

There are several novel formulations now a days which are used for the treatment of various diseases. But as an Emulgel act double role in this form and have more beneficial effects other than same categories formulations. Other than it various permeation enhancers can potentiate the effect, so Emulgel can be used as better topical drug delivery systems over present systems. The use of Emulgel can be expanded in different classification namely analgesics, antiinflammatory, antifungal, anti-acne drugs and various cosmetic formulation.

\section{Compliance with ethical standards}

\section{Acknowledgments}

The author Acknowledge Ms. Beena Kumari, Department of Pharmaceutical Sciences, IGU, Meerpur, Rewari, Haryana for her entire guidance and support.

\section{Disclosure of conflict of interest}

All authors declare that no conflict of interest is exist.

\section{References}

[1] Verma A, Singh S, Kaur R and Jain UK. (2013). Topical gels as drug delivery systems. International Journal of Pharmaceutical Sciences Review and Research, 23(2), 374-382.

[2] Jani R, Jani K, Setty CM and Dipti P. (2010). Preparation and evaluation of topical gel of Valdecoxib. International Journal of Pharmaceutical Sciences and Drug Research, 2(1), 51-54.

[3] Singla V, Saini S, Joshi B and Rana AC. (2012). Emulgel: A new platform for topical drug delivery. International Journal of Pharma and Biosciences, 3(1), 485-498.

[4] Dadwal M. (2013). Emulgel: A novel approach to topical drug delivery. International Journal of Pharma and Biosciences, 4(1), 847-856. 
[5] Pradeep, Kamal and Kumari B. (2020). Versatility of nanosuspension formulation in various drug delivery systems: A brief review. Advance Pharmaceutical Journal, 5(2), 36-46.

[6] Arora V, Kumar P and Sharma R. (2015). Emulgel: A review for topical drug delivery of hydrophobic drugs. International Journal of Pharma professional's Research, 6(3), 1256-1262.

[7] Swathi B, Kusuma G, Kusuma K and Kumar PM. (2013). Emulgel: As a topical drug delivery system. International Journal review life sciences, 3(3), 58-65.

[8] Hyma P, Jahan N, Raheemunissa, Sreelekha G and Babu K. (2014). Emulgel: A review. International Journal of Pharmaceutical Arachive, 3(3), 1-11.

[9] Mathew LK and Abraham S. (2014). Emulgel: An innovative approach for topical drug delivery. International Journal of Universal Pharmacy and Bio Sciences, 3(4), 207-223.

[10] Aher SD, Banerjee SK, Gadhave MV and Gaikawad DD. (2013). Emulgel: A new dosage form for topical drug delivery. International Journal of Institutional Pharmacy and Life Sciences, 3(3), 1-10.

[11] Vats S, Saxena C, Easwari TS and Shukla VK. (2014). Emulsion and gel technique: novel approach for enhancing topical drug delivery of hydrophobic drugs. International Journal for Pharmaceutical Research Scholars, 3(2), 649-660.

[12] Shah AA, Kamdar K, Shah R and Keraliya RA. (2013). Emulgel: A topical preparation for hydrophobic Drugs. Pharma Tech Medicines, 2(2), 370-375.

[13] Singh P, Sharma G, Bala R and Gill NS. (2015). Emulgel: An emerging technique for topical drug delivery system. International Journal of Recent Advances in Pharmaceutical Research, 5(1), 1-8.

[14] Ganesh TA, Dattatraya SM and Bhanudas. (2013). Hydrogel- A novel technique for preparation of topical gel. World Journal of Pharmacy and Pharmaceutical Sciences, 2(6), 4520-4541.

[15] Sharma N, Agarwal G, Rana Z, Bhat A and Kumar D. (2011). A review: transdermal drug delivery system: a tool for novel drug delivery system. International Journal of Drug Development and Research, 3(3), 70-84.

[16] Singla V, Saini S, Joshi B and Rana AC. (2012). Emulgel: A new platform for topical drug delivery. International Journal of Pharma and Biosciences, 3(1), 485-498.

[17] Joshi B, Singh G, Rana AC, Saini S and Singla V. (2011). Emulgel: A comprehensive Review on the recent advance in topical drug delivery. International Research Journal of Pharmacy, 2(11), 66-70.

[18] Panwar S, Mukhopandhay S and Kothiyal P. (2015). Emulgel: A novel approach for topical drug delivery system. International Journal of Pharmaceutical Research and Bio-Science, 4(4), 209-223.

[19] Mohamed MI. (2004). Optimization of Chlorphensin emulgel formulation. The AAPS Journal, 6(3), 1-6.

[20] Kumari B. (2020). Advances in Medical and Pharmaceutical Sciences. Ist Edition, ESN Publications, Chennai, India.

[21] Wani RR, Patil MP, dhurjad CA and Kshirsagar SJ. (2015). Microemulsion based gel: A novel approach in delivery of hydrophobic drugs. International Journal for Pharmaceutical Research Scholars, 4(2), 397-410.

[22] Asija R, Sharma R and Gupta A. (2013). Emulgel: A novel approach to topical drug delivery. Journal of Biomedical and Pharmaceutical Research, 2(6), 91-94.

[23] Eswaraiah S, Swetha K, Lohita M, Preethi J and Reddy KK. (2014). Emulgel: review on novel approach to topical drug delivery. International Journal of Pharmaceutical Research, 4(1), 4-11.

[24] Kokane V and Naik S. (2014). Formulation and evaluation of topical of Flurbiprofen gel using different gelling agents. World Journal of Pharmacy and Pharmaceutical Sciences, 3(9), 654-663.

[25] Kamal, Pradeep and Kumari B. (2020). Niosomes. In: Advances in Medical and Pharmaceutical Sciences. ESN Publications, Chennai, India, 51-64.

[26] Kitawat S, Saxena A and Gaur K. (2015). Formulation development and evaluation of Aceclofenac Sodium gel. Journal of Chemical and Pharmaceutical Research, 7(10), 948-952.

[27] Suvarnalata MS and Chaudhari RY. (2016). Transdermal gel: As a novel drug delivery system. International Journal of Pharmacy and Life Sciences, 7(1), 4864-4871.

[28] Patel J, Trivedi J and Chudhary S. (2014). Formulation and evaluation of Diacerin Emulgel for psoriatic arthritis. International Journal of Pharmaceutical Research, 3(2), 625-638. 
[29] Sangale PT and Gadhave MV. (2014). Organogel: A novel approavh for transdermal drug delivery system. World Journal of Pharmaceutical Research, 4(3), 423-442.

[30] Kumari B. (2018). A Review on Nanoparticles: Their Preparation method and applications. Indian Research Journal of Pharmacy and Science, 5(2), 1420-1426.

[31] Kaur L and Kaur P. (2014). Formulation and evaluation of topical gel of Meloxicam. International Journal of Research in Pharmacy and Chemistry, 4(3), 619-623.

[32] Shukr MH and Metwally GF. (2013). Evaluation of topical gel bases formulated with various essential oils for antibacterial activity against Methicillin resistant staphylococcus aureus. Tropical Journal of Pharmaceutical Research, 12(6), 877-884.

[33] Pant S, Badola A, Baluni S and Pant W. (2015). A review on Emulgel novel approach for topical drug delivery system. World Journal of Pharmacy and Pharmaceutical Sciences, 4(10), 1728-1743.

[34] Khullar R, Saini S, Seth N and Rana AC. (2011). Emulgel: A surrogate approach for topically used hydrophobic drug. International Journal of Pharmacy and Biological Sciences, 1(3), 117-128.

[35] Roychowdhury S, Singh DH, Gupta R and Manjit D. (2012). A review of pharmaceutical gel. International Journal of Pharmaceutical Research and Bioscience, 1(5), 21-36.

[36] Swetha CH, Sellappan V, Nagarjuna PR and Reddy GN. (2013). Formulation and evaluation of Clarithromycin topical gel. International Journal of Drug Development and research, 5(4), 194-202.

\section{How to cite this article}

Devi S, Sangeeta and Kumari B. (2020). Emugel for topical drug delivery: A novel approach. GSC Biological and Pharmaceutical Sciences, 11(3), 104-114. 